\title{
Lactobacillus casei MYL01 modulates the proinflammatory state induced by ethanol in an in vitro model
}

\author{
Yi-Heng Chiu, ${ }^{\star}$ Jaw-Ji Tsai, $†$ Shiao-Lin Lin, $\neq$ and Meei-Yn Lin*1 \\ *Department of Food Science and Biotechnology, National Chung Hsing University, Taichung 40227, Taiwan, Republic of China \\ †Department of Internal Medicine, Veterans General Hospital International Medical Service Centre, Taichung 40705, Republic of China \\ ‡Department of Neurology, Chong Guang Hospital, Miaoli County 35159, Taiwan, Republic of China
}

\section{ABSTRACT}

Accumulating studies have suggested that probiotics have beneficial effects on liver injury but the underlying mechanism has remained unclear. Toll-like receptors (TLR) expressed on immune cells and hepatocytes recognize bacterial components that are translocated from the gut into the portal vein. To date, it has been demonstrated that ethanol alone, without microbial components, is able to activate TLR, leading to promotion of proinflammatory cytokine production. Because the enhanced signaling of TLR triggers persistent inflammation, we hypothesized that development of hepatocyte TLR tolerance to repetitive stimulation plays an important role in protecting the liver from hypergeneration of proinflammatory cytokines. In this study, we showed that Lactobacillus casei MYL01 modulated the proinflammatory state induced by ethanol and investigated in detail the mechanism underlying the observation that $L$. casei MYL01 gave rise to TLR tolerance toward ethanol stimulation. The effects of L. casei MYL01 in the attenuation of ethanol-induced liver damage were due to enhancement of IL-10 production, which limited the proinflammatory process. Furthermore, better defense of hepatocytes against ethanol challenge by treatment of $L$. casei MYL01 was attributed to previous induction of toll interacting protein (TOLLIP) and suppressor of cytokine signaling $(S O C S) 1$ and SOCS3 expression via activation of TLR1, TLR2, TLR6, and TLR9, an action that cross-regulated ethanol-TLR4-nuclear factor $\kappa \mathrm{B}$ signal transduction events. This finding might help establish an in vitro platform for selecting hepatoprotective probiotic strains in terms of ethanol-induced liver damage. Key words: toll-like receptor, probiotic, ethanol, liver injury

Received September 21, 2013

Accepted December 15, 2013.

${ }^{1}$ Corresponding author: meeiynlin@gmail.com

\section{INTRODUCTION}

Recently, with an increase in molecular approaches to the study of the etiology of alcoholic liver disease (ALD), we have found several causes contributing to the pathogenesis of ALD. Factors contributing to liver injury or hepatotoxicity include irregular oxidative stress, increased proinflammatory cytokine secretions, and overactive innate immune responses caused by ethanol as well as its metabolites or derivatives (Shukla et al., 2013). Leclercq et al. (2012) reported that ethanol-administered animals are characterized by intestinal hyperpermeability (also called leaky gut) and increased proliferation of gram-negative bacteria in the gastrointestinal tract, a situation that gives rise to elevated levels of proinflammatory cytokines into blood circulation. Systemic exposure to endotoxins from gram-negative bacteria accompanied by elevation of IL-8 and tumor necrosis factor $\alpha$ (TNF- $\alpha$ ) has been recognized as representative features of ALD progression (Kopeć et al., 2013). Endotoxins are a family of molecules that bind to many pattern recognition receptors that are expressed by cells of the innate immune system to identify microbial components. One of the most dominant endotoxins is LPS.

Lipopolysaccharides are powerful proinflammatory stimuli that act on toll-like receptor (TLR)4; TLR4 recognizes LPS from gram-negative bacteria, playing a role in relaying signals to nuclear factor kappa-lightchain-enhancer of activated B cells (nuclear factor $\kappa \mathrm{B}$; NFkB), a transcription factor responsible for production of proinflammatory cytokines and chemokines (Lucas and Maes, 2013). Evidence has suggested that the development of ALD is not only due to ethanol toxicity but also to inflammatory processes induced by circulative LPS (Mandrekar and Szabo, 2009). Additionally, long-term ethanol consumption is thought to stimulate the classical complement pathway, resulting in chronic elevation of proinflammatory cytokine secretions that increase the risk of ALD (Wlazlo et al., 2013). In addition to the LPS-TLR4 pathway, several studies have noted that ethanol, even in the absence of LPS, is able to activate TLR4 signaling pathways. Pascual et al. 
(2011) pointed out that suppression of TLR4 abolishes the effects of ethanol on both the innate and the adaptive inflammatory response in macrophages. They concluded that modulation of TLR4 function might ameliorate alcohol-induced inflammation.

To date, many reports have suggested that probiotics have antioxidant ability and immunomodulation capacity and are able to decrease LPS-induced inflammation (Grompone et al., 2012). Numerous mechanisms of probiotic action on liver disease have been proposed, including maintenance of gut barrier functions, reduction of bacterial translocation into blood circulation, homeostatic regulation of the gut cells toward an antiinflammatory milieu, and attenuation of liver oxidative stress (Forsyth et al., 2009). Supporting the mechanism-based approach that reduction of oxidative stress, modulation of pro- or antiinflammatory cytokine homeostasis, and desensitization of hepatic inflammation responses is effective in ALD improvement, the approach raises the possibility that probiotics could exploit these favorable characteristics to prevent and improve alcohol-induced liver injury.

Engagement of TLR4 induces a signaling cascade, resulting in the activation of multiple genes and proteins involved in innate immune responses. Prior exposure to LPS results in TLR4 being less responsive to subsequent similar challenge, which is regarded as LPS tolerance (Nahid et al., 2013). Development of LPS tolerance has been linked to enhanced resistance and protection against cellular injuries and is attributed to poor signal transduction in the TLR4-NFkB pathway. Toll-like receptor 4 recruits specific combinations of signal transducers to exert specific immune responses adapted to infectious pathogens (TLR4- Myd88-IRAK1-TRAF6TAK1-IKK-NFאB). Once TLR4 detects LPS, myeloid differentiation primary response gene 88 (Myd88) acts as an adapter protein essential for triggering the downstream signal cascade. Interleukin-1 receptor-associated kinase 1 (IRAK1) is recruited close to Myd88, mediating the downstream signaling transduction induced by LPS. Then, TNF receptor-associated factor 6 (TRAF6) kinase mediates signal transduction from IRAK1, offering a connection between IRAK1 and mitogen-activated protein kinase 7 (TAK1); TAK1 belongs to the family of serine- or threonine-specific protein kinases responsible for phosphorylation of a variety of downstream targets. Upon activation, TAK1 in turn activates $\mathrm{I} \kappa \mathrm{B}$ kinase (IKK), an enzyme complex involved in phosphorylation of cytoplasmic I $\kappa \mathrm{B} \alpha$, the action that gives rise to $\mathrm{NF \kappa B}$ nuclear translocation, triggering synthesis of proinflammatory cytokines (Chanput et al., 2010; Morris and Li, 2012).

Over the past decades, several negative regulators that inhibit TLR4 signal transduction to $\mathrm{NF} \kappa \mathrm{B}$ through various pathways have been revealed. Suppressors of cytokine signaling 1 and 3 (SOCS1, SOCS3) are cytokine-inducible negative regulators of cytokine signaling. They play key roles in the suppression of cytokine receptors and take part in a TLR negative feedback loop to attenuate phosphorylation of cytoplasmic I $\kappa$ B $\alpha$ by IKK (Dalpke et al., 2008). Toll interacting protein (TOLLIP) is an inhibitory adaptor protein, effectively inhibiting the activity of IRAK1 after TLR4 activation. Excess expression of TOLLIP has been shown to inhibit inflammation in response to TLR4 signaling (Antosz and Choroszynska, 2013). Interleukin-1 receptor-associated kinase 3 (IRAK3) restrains the dissociation of IRAK1 from Myd88 and the connections among TRAF6 complexes, causing interference of downstream signal relaying (van Hemert et al., 2010). Phosphatidylinositol-3,4,5-trisphosphate 5-phosphatase 1 (SHIP1) hydrolyses phosphatidylinositol 3-kinase (PI3K), which is activated in response to LPS stimulation; SHIP1 plays a terminating role in the TLR4 signaling cascades triggered by LPS (Hazeki et al., 2007).

Because TLR4 can be activated by both LPS and ethanol, it is reasonable to postulate that substances able to induce LPS tolerance probably also induce tolerance to ethanol (Wang et al., 2013a). Because most probiotics are gram-positive bacteria, we hypothesized that Lactobacillus casei MYL01 would activate the receptors TLR1, TLR2, TLR6, and TLR9, rather than TLR4, and in turn induce TLR negative regulators, thus contributing to development of TLR4 tolerant to ethanol.

Based on the theoretical positions taken for the study and the status of the field as briefly reviewed above, this research aimed to provide an in vitro model to elucidate how $L$. casei MYL01 results in better defense of hepatocytes against ethanol challenge.

\section{MATERIALS AND METHODS}

\section{Cell Culture}

Human hepatocellular carcinoma cells (HepG2) were obtained from the Bioresource Collection and Research Centre (BCRC, Taiwan, ROC). HepG2 cells were grown in Dulbecco's modified Eagle's medium (DMEM) containing $10 \%$ heat-inactivated fetal bovine serum. Cells were cultured at $37^{\circ} \mathrm{C}$ in a humidified (95\%) atmosphere with $5 \% \mathrm{CO}_{2}$.

\section{Source of Bacterial Strain and Preparation of Heat-Killed Bacteria}

Isolation and identification of L. casei MYL01 from human breast milk was conducted in the Microbiol- 
ogy Laboratory of National Chung Hsing University, Taiwan. Lactobacillus casei MYL01 was cultured in de Man, Rogosa, and Sharpe broth (Difco Laboratories, Detroit, MI) at $37^{\circ} \mathrm{C}$ for $16 \mathrm{~h}$. The culture was adjusted to $1 \times 10^{7} \mathrm{cfu} / \mathrm{mL}$ and washed twice with PBS, followed by killing at $65^{\circ} \mathrm{C}$ for $30 \mathrm{~min}$.

\section{Determination of Optimal Co-Incubation Time of HepG2 Cells with Heat-Killed L. casei MYL01 and Ethanol}

Before co-incubation of HepG2 cells with heat-killed $L$. casei MYL01 and ethanol, we performed the [3-(4,5-dimethylthiazol-2-yl)-2,5 diphenyl tetrazolium bromide; MTT] assay to assess the most appropriate incubation time. HepG2 cells $\left(1 \times 10^{6}\right.$ cells $\left./ \mathrm{mL}\right)$ were plated onto each well of 96-well plates for $24 \mathrm{~h}$, followed by treatment with heat-killed L. casei MYL01 $\left(1 \times 10^{7} \mathrm{cfu} / \mathrm{mL}\right)$ or ethanol $(100 \mathrm{~m} M)$ separately for $6,8,10,12,14,16$, 18, 20, 22, 24, and $26 \mathrm{~h}$. After incubation, viability was evaluated according to the MTT assay kit manufacturer's instructions (Sigma-Aldrich, St. Louis, MO).

For cytokine secretion assessment, HepG2 cells (1 $\times 10^{6}$ cells $/ \mathrm{mL}$ ) were plated onto each well of 96 -well plates for $24 \mathrm{~h}$, followed by treatment with heat-killed L. casei MYL01 $\left(1 \times 10^{7} \mathrm{cfu} / \mathrm{mL}\right)$ for $20 \mathrm{~h}$. After stimulation, HepG2 cells were challenged with $100 \mathrm{mM}$ ethanol for $20 \mathrm{~h}$. The supernatants were assayed for IL-8, IL-10, and TNF- $\alpha$ by ELISA (eBioscience, San Diego, CA). All culture conditions were set at $37^{\circ} \mathrm{C}$ in a humidified (95\%) atmosphere with $5 \% \mathrm{CO}_{2}$.

\section{Small Interfering RNA Silencing Technique}

Knockdown of expression of human TLR1, TLR2, TLR6, TLR9, TOLLIP, SOCS1, and SOCS3 in HepG2 cells was achieved by siGENOME SMARTpool assay kit (Dharmacon, Lafayette, CO). Antisense oligonucleotides were transfected with DharmaFECT 4 reagent according to the manufacturer's instructions. The small interfering (si)RNA experiment was carried out for 48 $\mathrm{h}$, at which time the cells were harvested for total RNA to analyze the silencing effect by quantitative reverse transcription-PCR (RT-qPCR).

\section{$R T-q P C R$}

Purification of RNA was performed by using the EZ-RNA total RNA isolation kit (Biological Industries, Beit Haemek, Israel). Reverse transcription was conducted according to manufacturer's instruction (BioRad iScript cDNA synthesis kit, Bio-Rad Laboratories, Hercules, CA). Quantification of gene expression by $\mathrm{qPCR}$ was carried out adopting the following primer designs: SOCS3 (5'-CAA ATG TTG CTT CCC CCT TA-3' and 5'-ATC CTG GTG ACA TGC TCC TC-3'), SHIP1 (5'-TCC AGC AGT CTT CCT CAC CT- $3^{\prime}$ and 5'-GCT TGG ACA CCA TGT TGA TG-3'), IRAK3 (5'-GGG TGC CTG TAG CAG AGA AG-3' and 5'ATC TGG AGG AGC CAG GAT TT-3'), SOCS1 (5'CTG GGA TGC CGT GTT ATT TT-3' and 5'-TAG GAG GTG CGA GTT CAG GT-3'), TOLLIP (5'-CCA CAG TGT GAG GGA TTG TG-3' and 5'-TCT CCT TCT CAT GCC GTT CT-3'), TLR1 (5'-CTG CCA ATT GCT CAT TTG AA-3' and 5'-TCC AGC TGA CCC TGT AGC TT-3'), TLR2 (5'-AAC CGG AGA GAC TTT GCT CA-3' and 5'-AGT TAT TGC CAC CAG CTT CC-3'), TLR 4 (5'-TGA GCA GTC GTG CTG GTA TC-3' and 5'-CAG GGC TTT TCT GAG TCG TC-3'), TLR6 (5'-TTC CAG AGC TGC CAG AAG AT-3' and 5'-GAG ATA CCA GGG CAG ATC CA-3'), TLR9 (5'-CCT ATT CAT GGA CGG CAA CT-3' and 5'-GAT GTA GCT GGG GGA AGT GA$\left.3^{\prime}\right)$, and GAPDH (5'-GAG TCA ACG GAT TTG GTC GT-3' and 5'-TTG ATT TTG GAG GGA TCT CG$\left.3^{\prime}\right)$. After reverse transcription of mRNA, $5 \mu \mathrm{L}$ of the reverse transcription product were added to a BioRad iCycler PCR system containing a $0.3 \mu M$ concentration of each primer. One-fold QuantiTect SYBR Green PCR Master Mix was used as a fluorescent reporter (Qiagen, Hilden, Germany). The conditions were programmed as follows: (1) denaturation at $94^{\circ} \mathrm{C}$ for $10 \mathrm{~min}$; and (2) amplification for 40 cycles of denaturation at $94^{\circ} \mathrm{C}$ for $15 \mathrm{~s}$, annealing at $55^{\circ} \mathrm{C}$ for $30 \mathrm{~s}$, and extension at $72^{\circ} \mathrm{C}$ for $20 \mathrm{~s}$. Gene expression was normalized to that of $G A P D H$.

\section{Western Blotting}

Nuclear and cytoplasmic proteins were harvested using Nuclear/Cytosol Fractionation Kit (Biovision, Milpitas, CA). Samples (30 $\mu \mathrm{g}$ of protein per well) were subjected to SDS-PAGE and transferred onto polyvinylidene fluoride membranes. Target proteins were incubated with a 1:2,000 dilution of $\mathrm{I} \kappa \mathrm{B} \alpha$ rabbit polyclonal IgG (Merck Millipore, Darmstadt, Germany), p65 rabbit polyclonal IgG (Merck Millipore), and a 1:5,000 dilution of actin mouse monoclonal IgG (Merck Millipore) at room temperature for $2 \mathrm{~h}$. Results were detected by using the ECL chemiluminescence detection kit (Merck Millipore). Protein expressions were normalized by actin. Protein quantification comparisons were analyzed by ImageJ (National Institutes for Health, Bethesda, MD).

\section{Statistical Analysis}

Each value is presented as mean \pm standard deviation $(\mathrm{n}=3)$. Statistical analysis of viability assay was 
carried out by Student's t-test. Comparisons of gene and protein quantities were analyzed by ANOVA, followed by Tukey's range test; $P<0.05$ was considered significantly different. All data were analyzed with SPSS statistical software (SPSS Inc., Chicago, IL), and figures were generated with SigmaPlot (Systat Software Inc., San Jose, CA).

\section{RESULTS}

\section{L. casei MYL01 Modulates Ethanol-Induced Secretion of Proinflammatory Cytokines}

Before co-incubation of HepG2 cells and heat-killed L. casei MYL01, we performed the MTT assay to assess the most appropriate incubation time. Results indicated that cell viability was not influenced significantly within $24 \mathrm{~h}$, and showed that a 20-h incubation time gave the maximum mean of viability (Figure 1). As a consequence, we cocultured HepG2 cells with heatkilled L. casei MYL01 or ethanol for $20 \mathrm{~h}$ in the following experiment.

As Figure 2 shows, heat-killed L. casei MYL01 showed excellent potency in terms of refractoriness to subsequent ethanol stimulation. Secretions of TNF- $\alpha$ and IL-8 decreased dramatically in the heat-killed $L$. casei MYL01 pretreatment group compared with the ethanol challenge group. In addition, we observed that both groups (heat-killed L. casei MYL01 alone and heat-killed L. casei MYL01 pretreatment before ethanol challenge) promoted production of the antiinflammatory cytokine IL-10. Heat-killed L. casei MYL01 also stimulated proinflammatory cytokine production but to a lesser degree. In addition to cytokine analysis, nuclear translocation of NFkB (a key transcription factor of pro-inflammatory cytokines) was assessed. As shown in Figure 3, exposure to ethanol had a profound effect on triggering p65 nuclear translocation, whereas exposure to heat-killed $L$. casei MYL01 only slightly induced p65 translocation. This consequence of NFKB translocation was in accordance with that of proinflammatory cytokine analysis, showing that ethanol challenge caused severe inflammation whereas heat-killed $L$. casei MYL01 induced inflammation to a mild degree.

\section{L. casei MYL01 and Ethanol-Activated Expression of TLR Genes}

To further explore the underlying mechanisms of ethanol tolerance development in HepG2 cells, RT-qP$\mathrm{CR}$ was conducted and the results are shown in Figure 4. The gene expression of TLR1, TLR2, TLR6, and TLR 9 was increased in the heat-killed L. casei MYL01 treatment group compared with the ethanol and nega-

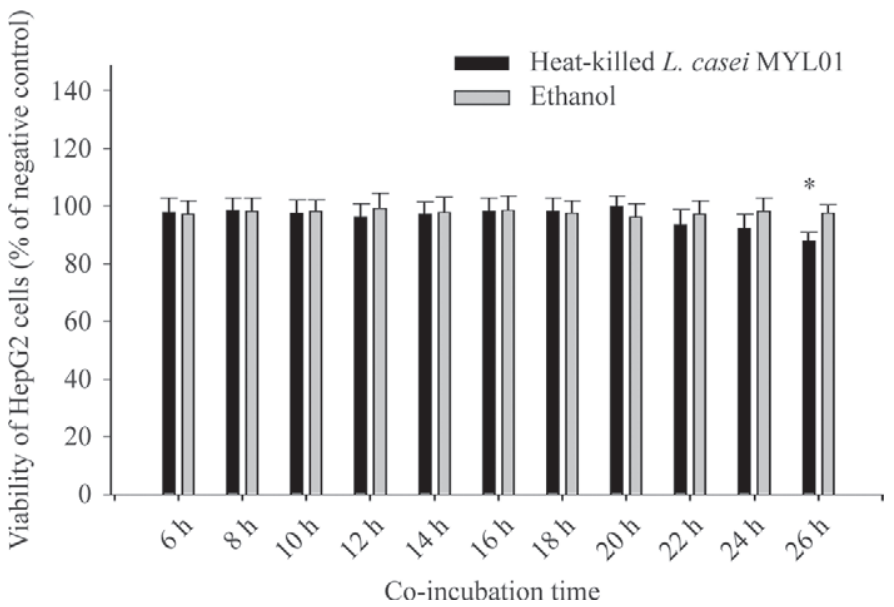

Figure 1. Viability of HepG2 cells in different co-incubation times: relative cell viability was expressed as the mean percentage of that of negative control (untreated HepG2 cells). Cells were cultured at $37^{\circ} \mathrm{C}$ in a humidified $(95 \%)$ atmosphere with $5 \% \mathrm{CO}_{2}$. ${ }^{*}$ indicates $P<0.05$ as assayed by Student's $t$-test $(\mathrm{n}=3)$.

tive control groups. However, expression of TLR 4 was unchanged in the heat-killed $L$. casei MYL01 group but enhanced in the ethanol challenge group.

\section{L. casei MYL01 Induces Expression of TOLLIP and SOCS1/3}

To illuminate the relative contributions of TLR negative regulators, 5 potential negative regulator gene

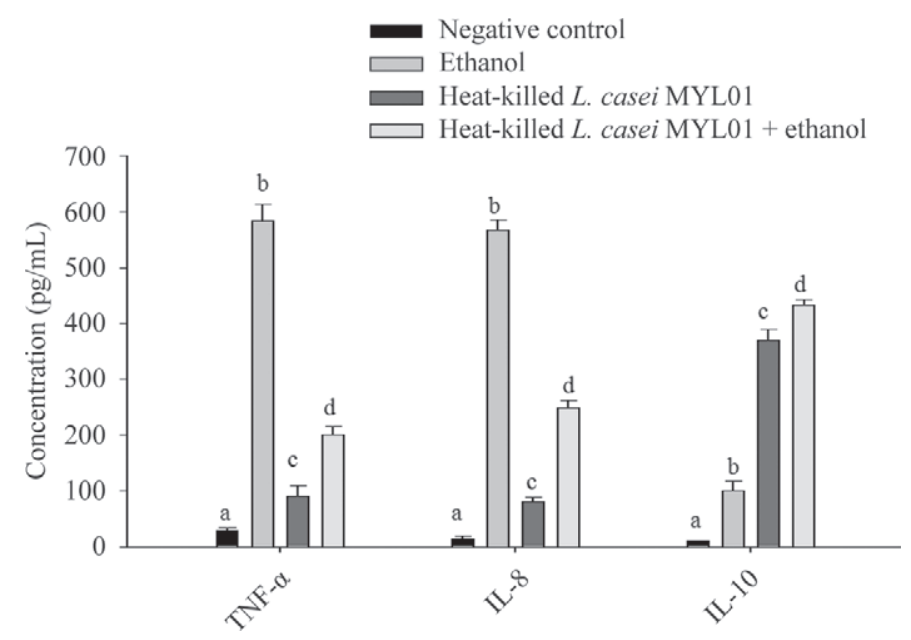

Figure 2. Cytokine stimulation assay: HepG2 cells $\left(1 \times 10^{6}\right.$ cells/ $\mathrm{mL})$ were treated with heat-killed Lactobacillus casei MYL01 $\left(1 \times 10^{7}\right.$ $\mathrm{cfu} / \mathrm{mL}$ ) for $20 \mathrm{~h}$. After stimulation, HepG2 cells were challenged with $100 \mathrm{mM}$ ethanol for $20 \mathrm{~h}$. Cells were cultured at $37^{\circ} \mathrm{C}$ in a humidified (95\%) atmosphere with $5 \% \mathrm{CO}_{2}$. Negative control: HepG2 cells were treated with Dulbecco's modified Eagle's medium instead of ethanol or heat-killed L. casei MYL01. Statistical comparisons were carried out by ANOVA, followed by Tukey's range test. Different letters represent $P$-value $<0.05$ compared with negative control group $(\mathrm{n}=3)$. 


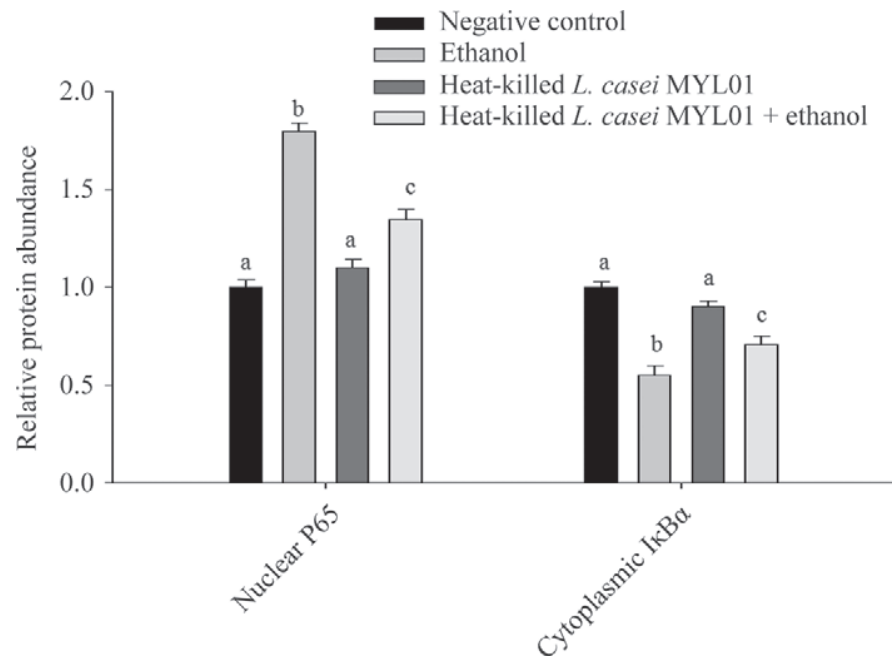

Figure 3. Nuclear factor- $\kappa \mathrm{B}(\mathrm{NF} \kappa \mathrm{B})$ translocation assay: heatkilled Lactobacillus casei MYL01 and ethanol challenge for $20 \mathrm{~h}$ af-

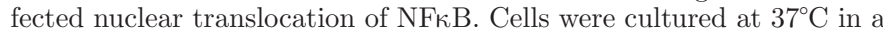
humidified (95\%) atmosphere with $5 \% \mathrm{CO}_{2}$. Negative control: HepG2 cells were treated with Dulbecco's modified Eagle's medium instead of ethanol or heat-killed L. casei MYL01. Statistical comparisons were carried out by ANOVA, followed by Tukey's range test. Different letters represent $P$-value $<0.05$ compared with negative control group $(\mathrm{n}=3)$.

expressions were assayed. As shown in Figure 5, we observed no considerable differences in the expression of IRAK3 and SHIP1 between groups. Although ethanol challenge gave rise to increased $S O C S 1 / 3$ expression

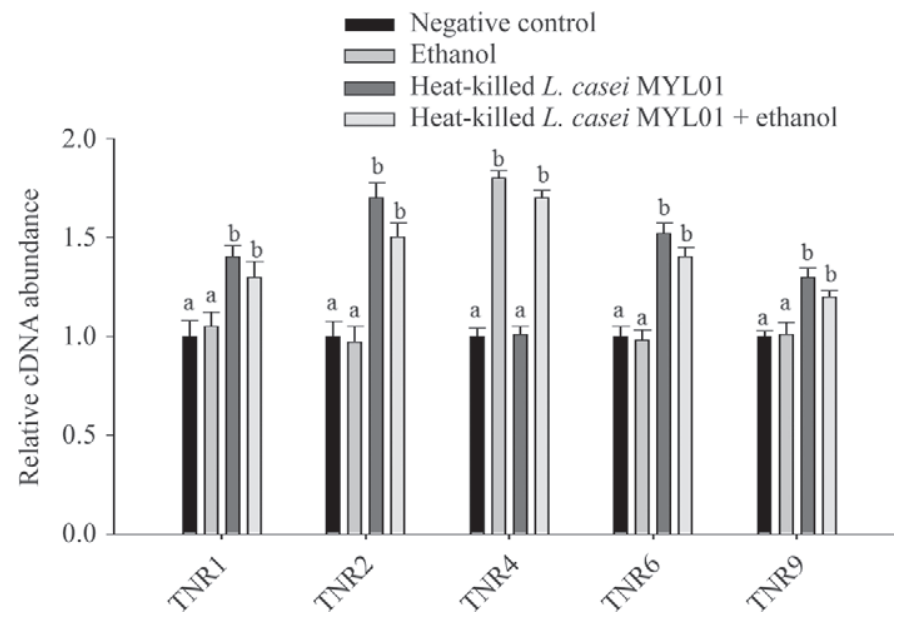

Figure 4. Toll-like receptor (TLR) stimulation ability of heatkilled Lactobacillus casei MYL01: HepG2 cells $\left(1 \times 10^{6}\right.$ cells $\left./ \mathrm{mL}\right)$ were treated with heat-killed $L$. casei MYL01 $\left(1 \times 10^{7} \mathrm{cfu} / \mathrm{mL}\right)$ and 100 $\mathrm{m} M$ ethanol for $20 \mathrm{~h}$, separately. Cells were cultured at $37^{\circ} \mathrm{C}$ in a humidified (95\%) atmosphere with $5 \% \mathrm{CO}_{2}$. Negative control: HepG2 cells were treated with Dulbecco's modified Eagle's medium instead of ethanol or heat-killed L. casei MYL01. Statistical comparisons were carried out by ANOVA, followed by Tukey's range test. Different letters represent $P$-value $<0.05$ compared with negative control group $(\mathrm{n}=3)$. compared with that of the negative control group, heatkilled $L$. casei MYL01 activated $S O C S 1 / 3$ to a greater extent (quantitatively) than did the ethanol group. Additionally, heat-killed L. casei MYL01 activated expression of TOLLIP, the negative regulator that was not significantly activated by ethanol challenge compared with the negative control group.

\section{Silencing of TLR Decreases Levels of TOLLIP and SOCS1/3 Genes}

We used a gene knockdown technique to silence expression of TLR1, TLR2, TLR6, and TLR9, which was induced by heat-killed L. casei MYL01 and ethanol, to address the relation between heat-killed $L$. casei MYL01 and TLR negative regulators. Preliminary tests revealed that silencing of target genes did not decrease the expression of nontarget genes significantly (data not shown). The TLR1, TLR2, TLR4, TLR6, and TLR9 genes were silenced separately and subsequently challenged with ethanol. Figure 6 shows that knockdown of TLR1, TLR2, TLR6, and TLR9 led to heat-killed $L$. casei MYL01, in part, failing to induce expression of TOLLIP (Figure 6A), SOCS1 (Figure 6B), or SOCS3 (Figure 6C); silencing of TLR2 decreased the expression of TLR negative regulators to the greatest degree. Of all the TLR, knockdown of TLR4 had less suppressive effects on the induction of SOCS1/3 and TOLLIP.

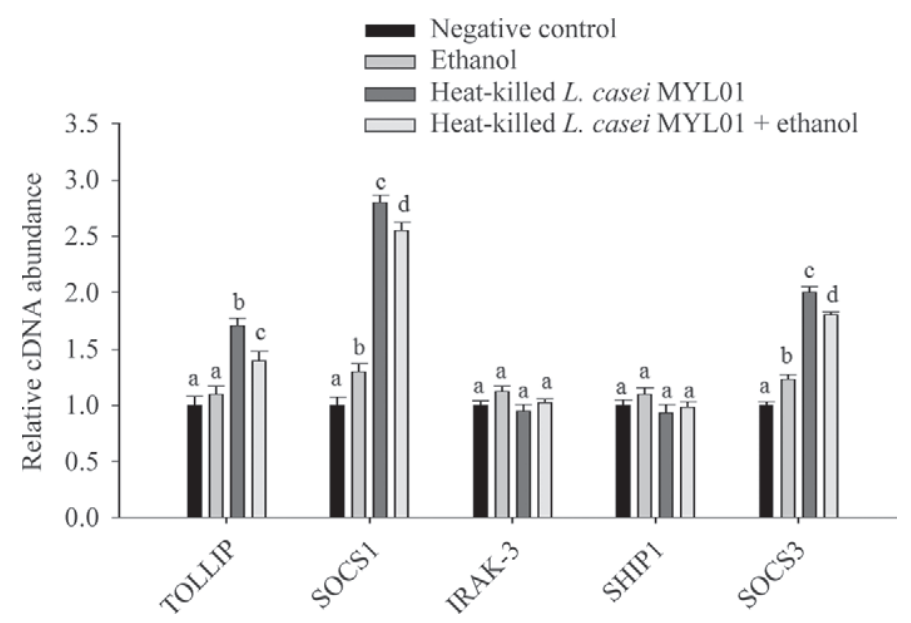

Figure 5. Toll-like receptor (TLR) negative regulator stimulation ability of heat-killed Lactobacillus casei MYL01: heat-killed L. casei MYL01 and ethanol stimulation for $20 \mathrm{~h}$ affected gene expressions of TLR negative regulators. Cells were cultured at $37^{\circ} \mathrm{C}$ in a humidified (95\%) atmosphere with $5 \% \mathrm{CO}_{2}$. Statistical comparisons were carried out by ANOVA, followed by Tukey's range test. Different letters represent $P$-value $<0.05$ compared with negative control group $(\mathrm{n}=3)$. 

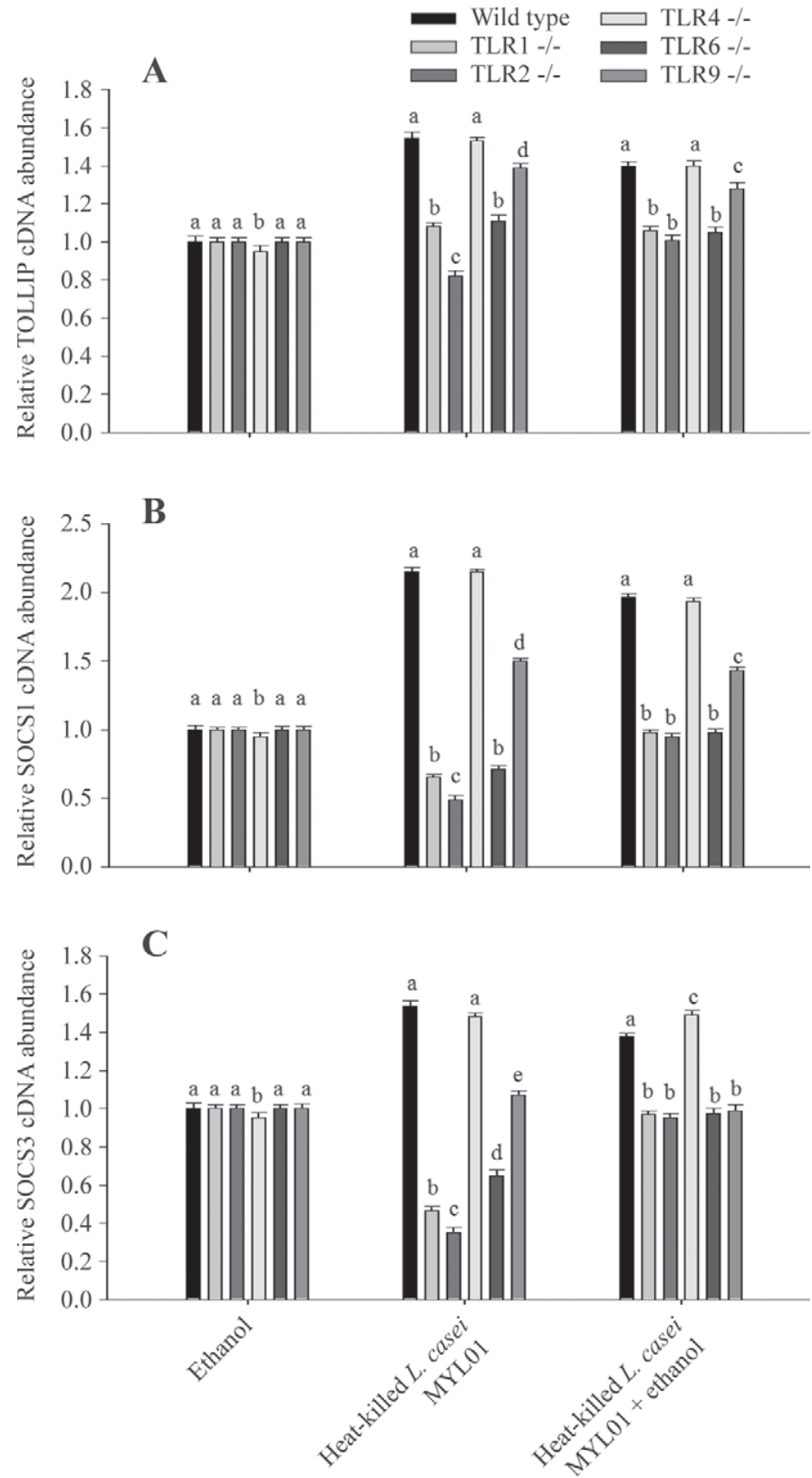

Figure 6. Knockdown of toll-like receptors (TLR) affect TLR negative regulator stimulation ability: HepG2 cells silenced by TLR1, TLR2, TLR4, TLR6, and TLR9 were treated with heat-killed Lactobacillus casei MYL01 for $20 \mathrm{~h}$ and expressions of toll interacting protein (TOLLIP; A), suppressor of cytokine signaling 1 (SOCS1; B) and suppressor of cytokine signaling 3 (SOCS3; C) were analyzed. Cells were cultured at $37^{\circ} \mathrm{C}$ in a humidified (95\%) atmosphere with $5 \%$ $\mathrm{CO}_{2}$. Statistical comparisons were carried out by ANOVA, followed by Tukey's range test. Different letters represent $P$-value $<0.05$ compared with negative control group $(\mathrm{n}=3)$.

\section{Knockdown of TOLLIP and SOCS1/3 Partly Abolishes the Antiinflammatory Ability of Heat-Killed L. casei MYLO1}

Effects of cytokine secretions upon TOLLIP and SOCS1/3 knockdown are presented in Figure 7. Knock- down of SOCS1 had the greatest effect on the antiinflammatory properties of heat-killed $L$. casei MYL01, whereas silencing of TOLLIP apparently did not influence the development of tolerance to the same degree as silencing of $S O C S 1 / 3$. This result corresponded with that of Figure 5, which showed gene expression levels activated by heat-killed $L$. casei MYL01 ranked by quantity as follows: SOCS1 $>$ SOCS3 $>$ TOLLIP. It is worth noting that IL-10 secretion of TOLLIP-, SOCS1- and SOCS3-silenced HepG2 cells showed more significant decreases than that of unsilenced HepG2 cells. Briefly, knockdown of TOLLIP and SOCS1/3 largely abolished the capacity of heat-killed L. casei MYL01 to induce IL-10.

\section{DISCUSSION}

The last few decades have seen growing importance placed on research into the effects of probiotics on liver protection. Forsyth et al. (2009) reported that Lactobacillus rhamnosus GG could reduce hepatic inflammation in a rat model of alcoholic steatohepatitis. Similar results were reported by Wang et al. (2013b); their research team proposed that $L$. rhamnosus GG ameliorates chronic alcohol-induced liver injury by improving intestinal oxidative stress and gut leakiness.

The effectiveness of probiotic use in diseases is usually evaluated during practical clinical trials; however, such trials have not shown high repeatability. Unconvincing and disappointing consequences may be attributed to the methods of probiotic screening, which are principally on the basis of resistance to artificial gastrointestinal conditions (acid and bile challenges) or intestinal cell adherence properties, rather than immunomodulation capacities; for instance, induction of endotoxin tolerance. To our knowledge, relatively little research has probed the area of probiotic-induced cross-tolerance of TLR4 and other TLR in liver cells. Therefore, we investigated the mechanism by which probiotics exert hepatoprotective effects from the aspect of TLR crosstolerance development.

It has been reported that Lactobacillus casei MYL01 modulated LPS-stimulated inflammation through

cross-regulation of TLR4 and NOD2 (nucleotidebinding oligomerization domain-containing protein 2) signaling in terms of LPS-induced liver injury (Chiu et al., 2013). Nevertheless, whether or not L. casei MYL01 could exploit these favorable characteristics to improve ethanol-induced liver injury remains unclear.

Within the in vitro study of HepG2, we investigated the development of TLR4 tolerance by heat-killed $L$. casei MYL01 in terms of decreased secretion of proinflammatory cytokines in response to restimulation by ethanol. We first examined the extent to which the 


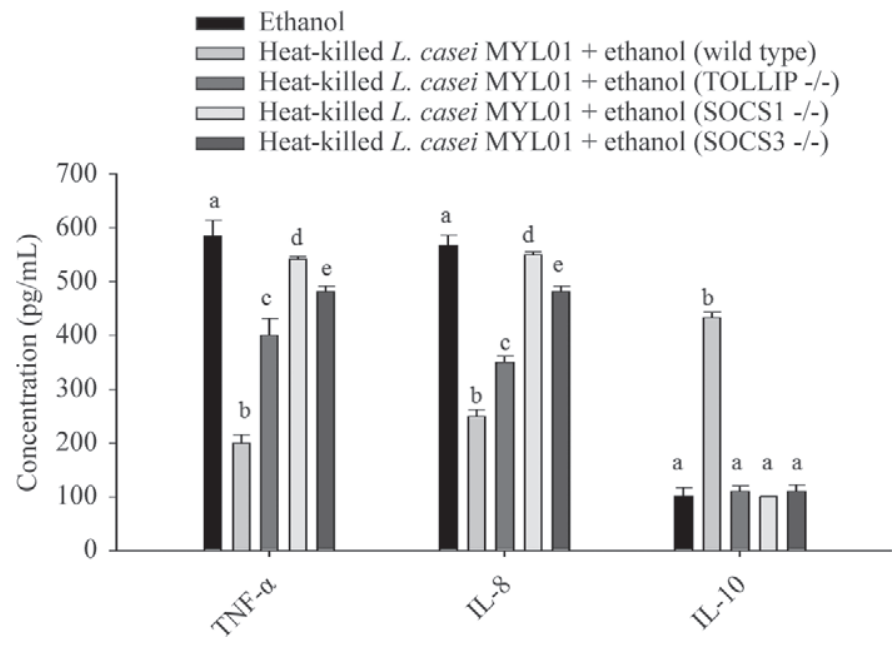

Figure 7. Knockdown of toll-like receptor (TLR) negative regulators affect cytokine secretions: HepG2 cells silenced by toll interacting protein (TOLLIP), suppressor of cytokine signaling $(S O C S) 1$ and SOCS3 were treated with heat-killed Lactobacillus casei MYL01 followed by ethanol challenge. Cells were cultured at $37^{\circ} \mathrm{C}$ in a humidified $(95 \%)$ atmosphere with $5 \% \mathrm{CO}_{2}$. Statistical comparisons were carried out by ANOVA, followed by Tukey's range test. Different letters represent $P$-value $<0.05$ compared with negative control group $(\mathrm{n}=3)$.

practical potency of TLR 4 tolerance was induced by $L$. casei MYL01. The consequence that pretreatment of HepG2 cells with heat-killed $L$. casei MYL01 effectively modulated TNF- $\alpha$ and IL- 8 production in response to ethanol challenge validated the precondition for subsequent exploration of the molecular mechanism.

It is known that cellular components of probiotics are recognized by pattern recognition receptors, such as the NOD-like receptors (NLR) and the TLR family. Downstream intracellular signaling from these receptors leads to modulation of cytokine profiles, but the mechanism of desensitization of these receptors is still controversial. It has been reported that hyporesponses of TLR2 (Smelt et al., 2013) and TLR9 (Hiramatsu et al., 2013) could be involved in antiinflammatory effects associated with some strains of probiotics. However, one of the difficulties researchers face is that the capacities for stimulating pattern recognition receptors vary from species to species and even from strain to strain. We investigated the expression of TLR1, TLR2, TLR4, $T L R 6$, and TLR 9 after challenge of ethanol and heatkilled L. casei MYL01, and found that TLR4 expression was induced by ethanol but not by heat-killed $L$. casei MYL01. The result was in line with extensive literature reporting that TLR4 does not react with gram-positive bacteria (McCarthy et al., 2014). To a design more reliable and objective approach, we assayed transcription factors of proinflammatory cytokines to prove that heat-killed L. casei MYL01 was unable to induce inflammation as severe as ethanol challenge. Considering the result of negligible nuclear translocation of NF- $\mathrm{B}$ p65 and enhanced quantity of cytoplasmic $I \kappa \mathrm{B} \alpha$, we further confirmed that activation of TLR1, TLR2, TLR6, and TLR 9 induced by heat-killed L. casei MYL01 did not bring about severe inflammation. This may be attributed to 2 possible factors. First, heat-killed $L$. casei MYL01 enhanced IL-10 production, which antagonized the proinflammatory process induced by TNF- $\alpha$ and IL-8 (Carey et al., 2012). Second, heat-killed L. casei MYL01 affected TLR-NFKB signaling negative regulators (Yamamoto and Takeda, 2010; Zhu and Mohan, 2010; Ramgolam and Markovic-Plese, 2011).

The results of Figure 2 support the view that enhanced IL-10 production was correlated with antiinflammatory properties. Pretreatment of heat-killed $L$. casei MYL01 enhanced IL-10 production, and therefore ethanol rechallenge did not lead to inflammation as severe as that of the ethanol challenge group. To support the second proposed mechanism, TLR-NFkB negative regulators including TOLLIP, SOCS1, SOCS3, IRAK3, and SHIP1 were examined to determine their involvement in downregulation of TLR $-\mathrm{NF} \kappa \mathrm{B}$ signaling. The findings from this study indicated that expression of TOLLIP and SOCS1/3 was enhanced by heat-killed $L$. casei MYL01 treatment, whereas expression of IRAK3 and SHIP1 was not.

Research on the development of TLR cross-tolerance addresses several questions that must be answered before we can conclude that TLR activations were related to the decreased inflammation and expression of TLR negative regulators activated by heat-killed $L$. casei MYL01. Hence, we conducted an siRNA technique for knockdown of TLR (Figure 6) and TLR negative regulators (Figure 7) to determine if TLR activation contributed to enhanced expression of TLR negative regulators, thus resulting in antiinflammation phenotype in HepG2 cells. Clearly, knockdowns of TLR1, TLR2, TLR6, and TLR9 abolished the antiinflammatory effects of heat-killed L. casei MYL01, whereas knockdown of TLR4 did not influence expression of TLR negative regulators TOLLIP and SOCS1/3. In other words, heat-killed $L$. casei MYL01 activated TLR negative regulators through TLR1, TLR2, TLR6, and TLR 9 but not through TLR 4Our results not only support the hypothesis that heat-killed L. casei MYL01 brought about negative feedback inhibition of ethanolinduced TLR4 signaling, but also imply that TLR1, TLR2, TLR6, and TLR 9 activated by heat-killed $L$. casei MYL01 were essential, to different degrees, for induction of TOLLIP and SOCS1/3. In conclusion, in an in vitro model, we suggest that the mechanisms by which L. casei MYL01 attenuates ethanol-induced liver damage were 2-fold. First, promotion of IL-10 secretion contributes to antagonization of proinflammatory cyto- 
kines. Second, prior induction of TOLLIP and SOCS1/3 expression via activation of TLR1, TLR2, TLR6, and TLR9 downregulates the ethanol-TLR4-NFkB signal transduction events.

\section{ACKNOWLEDGMENTS}

Appreciation is given to C. D. Chung (Saint Hawk Enterprise Inc., New Taipei City, Taiwan, ROC), for helpful discussion in this study.

\section{REFERENCES}

Antosz, H., and D. Choroszynska. 2013. [Negative regulation of tolllike receptor signalling]. Postepy. Hig. Med. Dosw. (Online) 67:339-350. (In Polish).

Carey, A. J., C. K. Tan, and G. C. Ulett. 2012. Infection-induced IL10 and JAK-STAT: A review of the molecular circuitry controlling immune hyperactivity in response to pathogenic microbes. JAKSTAT 1:159-167.

Chanput, W., J. Mes, R. A. M. Vreeburg, H. F. J. Savelkoul, and H. J. Wichers. 2010. Transcription profiles of LPS-stimulated THP-1 monocytes and macrophages: A tool to study inflammation modulating effects of food-derived compounds. Food Funct. 1:254-261.

Chiu, Y. H., S. L. Lin, C. C. Ou, Y. C. Lu, H. Y. Huang, and M. Y. Lin. 2013. Anti-inflammatory effect of lactobacilli bacteria on HepG2 cells is through cross-regulation of TLR4 and NOD2 signalling. J. Funct. Foods 5:820-828.

Dalpke, A., K. Heeg, H. Bartz, and A. Baetz. 2008. Regulation of innate immunity by suppressor of cytokine signaling (SOCS) proteins. Immunobiology 213:225-235.

Forsyth, C. B., A. Farhadi, S. M. Jakate, Y. Tang, M. Shaikh, and A. Keshavarzian. 2009. Lactobacillus GG treatment ameliorates alcohol-induced intestinal oxidative stress, gut leakiness, and liver injury in a rat model of alcoholic steatohepatitis. Alcohol 43:163172.

Grompone, G., P. Martorell, S. Llopis, N. Gonzalez, S. Genoves, A. P. Mulet, T. Fernandez-Calero, I. Tiscornia, M. Bollati-Fogolin, I. Chambaud, B. Foligne, A. Montserrat, and D. Ramon. 2012. Anti-inflammatory Lactobacillus rhamnosus CNCM I-3690 strain protects against oxidative stress and increases lifespan in Caenorhabditis elegans. PLoS ONE 7:e52493.

Hazeki, K., K. Nigorikawa, and O. Hazeki. 2007. Role of phosphoinositide 3-kinase in innate immunity. Biol. Pharm. Bull. 30:1617-1623.

Hiramatsu, Y., T. Satho, K. Irie, S. Shiimura, T. Okuno, T. Sharmin, S. Uyeda, Y. Fukumitsu, Y. Nakashima, F. Miake, and N. Kashige. 2013. Differences in TLR9-dependent inhibitory effects of $\mathrm{H}(2) \mathrm{O}(2)$-induced IL-8 secretion and NF-kappaB/I kappa B-alpha system activation by genomic DNA from five Lactobacillus species. Microbes Infect. 15:96-104.

Kopeć, A., E. Piątkowska, T. Leszczyńska, and A. Koronowicz. 2013. Effect of long term administration of resveratrol on lipid concentration in selected organs and liver's histology in rats fed high fructose diet. J. Funct. Foods 5:299-305.
Leclercq, S., P. D. Cani, A. M. Neyrinck, P. Starkel, F. Jamar, M. Mikolajczak, N. M. Delzenne, and P. de Timary. 2012. Role of intestinal permeability and inflammation in the biological and behavioral control of alcohol-dependent subjects. Brain Behav. Immun. 26:911-918.

Lucas, K., and M. Maes. 2013. Role of the toll like receptor (TLR) radical cycle in chronic inflammation: Possible treatments targeting the TLR4 pathway. Mol. Neurobiol. 48:190-204.

Mandrekar, P., and G. Szabo. 2009. Signalling pathways in alcoholinduced liver inflammation. J. Hepatol. 50:1258-1266.

McCarthy, C. G., S. Goulopoulou, C. F. Wenceslau, K. M. Spitler, T. Matsumoto, and R. C. Webb. 2014. Toll-like receptors and damage-associated molecular patterns: Novel links between inflammation and hypertension. Am. J. Physiol. Heart Circ. Physiol. 306:H184-H196. http://dx.doi.org/10.1152/ajpheart.00328.2013.

Morris, M., and L. Li. 2012. Molecular mechanisms and pathological consequences of endotoxin tolerance and priming. Arch. Immunol. Ther. Exp. (Warsz.) 60:13-18.

Nahid, M. A., B. Yao, P. R. Dominguez-Gutierrez, L. Kesavalu, M. Satoh, and E. K. Chan. 2013. Regulation of TLR2-mediated tolerance and cross-tolerance through IRAK4 modulation by miR-132 and miR-212. J. Immunol. 190:1250-1263.

Pascual, M., S. Fernandez-Lizarbe, and C. Guerri. 2011. Role of TLR4 in ethanol effects on innate and adaptive immune responses in peritoneal macrophages. Immunol. Cell Biol. 89:716-727.

Ramgolam, V. S., and S. Markovic-Plese. 2011. Regulation of suppressors of cytokine signaling as a therapeutic approach in autoimmune diseases, with an emphasis on multiple sclerosis. J. Signal Transduct. 2011:635721.

Shukla, S. D., S. B. Pruett, G. Szabo, and G. E. Arteel. 2013. Binge ethanol and liver: New molecular developments. Alcohol. Clin. Exp. Res. 37:550-557.

Smelt, M. J., B. J. de Haan, P. A. Bron, I. van Swam, M. Meijerink, J. M. Wells, M. Kleerebezem, M. M. Faas, and P. de Vos. 2013 The impact of Lactobacillus plantarum WCFS1 teichoic acid Dalanylation on the generation of effector and regulatory T-cells in healthy mice. PLoS ONE 8:e63099.

van Hemert, S., M. Meijerink, D. Molenaar, P. A. Bron, P. de Vos, M. Kleerebezem, J. M. Wells, and M. L. Marco. 2010. Identification of Lactobacillus plantarum genes modulating the cytokine response of human peripheral blood mononuclear cells. BMC Microbiol. 10:293.

Wang, Y., Y. Hu, C. Chao, M. Yuksel, I. Colle, R. A. Flavell, Y. Ma H. Yan, and L. Wen. 2013a. Role of IRAK-M in alcohol induced liver injury. PLoS ONE 8:e57085.

Wang, Y., Y. Liu, I. Kirpich, Z. Ma, C. Wang, M. Zhang, J. Suttles, C. McClain, and W. Feng. 2013b. Lactobacillus rhamnosus GG reduces hepatic TNFalpha production and inflammation in chronic alcohol-induced liver injury. J. Nutr. Biochem. 24:1609-1615.

Wlazlo, N., M. M. van Greevenbroek, I. Ferreira, E. H. Jansen, E. J. Feskens, C. J. van der Kallen, C. G. Schalkwijk, B. Bravenboer, and C. D. Stehouwer. 2013. Activated complement factor 3 is associated with liver fat and liver enzymes: The CODAM study. Eur. J. Clin. Invest. 43:679-688.

Yamamoto, M., and K. Takeda. 2010. Current views of toll-like receptor signaling pathways. Gastroenterol. Res. Pract. 2010:240365.

Zhu, J., and C. Mohan. 2010. Toll-like receptor signaling pathwaysTherapeutic opportunities. Mediators Inflamm. 2010:781235. 